\title{
Selecting the correct research method
}

\author{
Sarath Lekamwasam ${ }^{1}$ \\ Sri Lanka Journal of Diabetes, Endocrinology and Metabolism 2013; 3: 101-103
}

There are many methods or designs available for researches when conducting research. Same research question or hypothesis can be tested by different methods but the validity and applicability of results vary. Selecting the most appropriate research method to test the research question is an impotent and critical step in conducting a research.

Research methods, broadly, are of two types; observational and interventional. In each method there are two key elements; Exposure and Outcome. "Exposure" refers to the situation that investigator believes may have caused or may lead to the condition or disease in question (such as smoking or taking a drug). The word "Outcome" denotes the end result, i.e. the clinical condition or disease in question. Most studies basically examine the relationship between the exposure and the outcome. In addition, it can determine the direction and the strength of the relationship too.

The fundamental difference between observational and interventional studies is related to exposure. In observational studies researcher or investigator does not determine the exposure. He has no role to play in determining who would get exposed, the dose or duration of the exposure (e.g. smoking and alcohol consumption). In interventional studies investigator determines the types of people who would get the exposure, dose and duration (e.g. drug trial). As investigator does not determine the exposure it is relatively easy to get ethical approval for the observational studies. In interventional studies the investigator has to justify the exposure and other things related to it such as dose and duration and finally convince the authorities.

\section{Study designs}

1) Observational
a. Cohorts
b. Case control
c. Cross sectional

2) Interventional (experimental)
a. Clinical trials
b. Community trials

All study designs are not of same quality and they can be arranged in hierarchical manner (Figure 1). Designs in the top of the pyramid generate more valid information than those at the bottom. This is mainly due to the confounders and various forms of biases associated with the study designs at the bottom of the pyramid. Study designs at the top of the pyramid such as clinical trials make maximum effort to eliminate confounders and biases that can compromise the validity of the results.

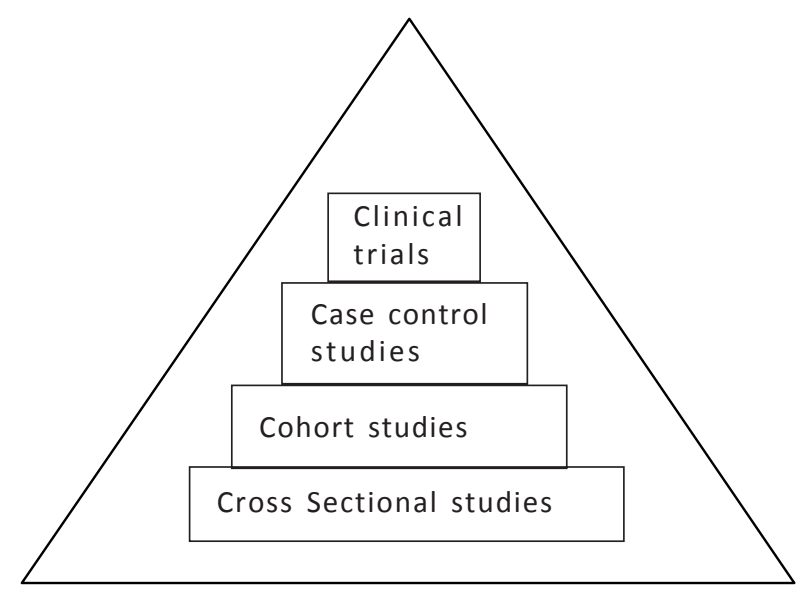

Figure 1. Hierarchical arrangement of research designs.

Case histories and case series, although not pure research, have generated valuable and clinically relevant information in the past. There are numerous landmark case histories that have prompted clinicians to think differently, pay more attention to changing disease patterns and inform about rare but serious adverse effects of drugs.

\section{Cohort studies}

Cohort studies recruit subjects based on the exposure and they are followed up until the desired outcome is manifested. As exposure and outcome are chronologically separated (no outcome at the time of recruitment) the association between them can be easily understood. 


\section{EXPOSURE $\longrightarrow$ OUTCOME}

\section{Examples}

1) Recruit all males in a town and separate them according to smoking habits and follow them up to detect the occurrence of bronchial carcinoma.

2) Recruit all women with first pregnancy in the first trimester in one $\mathrm{MOH}$ area and separate them according to body iron status and follow up to determine the birth weight of newborns.

Cohort studies have an advantage that multiple outcomes can be assessed. e.g. in addition to bronchial carcinoma in the 1st example, one can detect the occurrence of ischemic heart disease and bladder carcinoma etc. The major setback of the design is the period of follow up that may be required in some instances. Malignancies and myocardial ischemia may need several years (or decades) of exposure and study may require long period of follow up. This makes the study expensive, requires more time of the investigator and leads to more drop outs. People may change the residence or lose the interest of the study and may no longer available. Studies of shorter duration such as one described in the 2nd example eliminate some of these practical issues.

Despite all practical limitations cohort studies have contributed to the medical field enormously. Clear examples include Framingham and Rotterdam studies.

\section{Case control studies}

In contrast to cohort studies, case control studies recruit subjects based on the outcome. Cases are the subjects with the manifested outcome and they need to be matched with a group of subjects without the outcome. Once the two groups are found, investigator inquires about the exposure in all subjects.

\section{EXPOSURE}

\section{OUTCOME}

\section{Examples}

1) Recruit a group of patients with bronchial carcinoma and select an age and sex matched control group without bronchial carcinoma. Investigator then inquires about the smoking habits in the past.

2) Recruit a group of mothers who delivered babies with congenital cardiac defects and match with a group of mothers who delivered normal babies. Investigator then inquires about the infections that occurred and drugs they received in the first trimester.
Main advantage of the case control design is that it provides an opportunity to study the association between the exposure and outcome when they are separated by a long time gap (smoking and bronchial carcinoma in the 1st e.g.). One setback of the design is the limitations in recall of events that occurred long time ago. This, however, is common to both groups and may not cause a huge discrepancy of data in the two groups. More serious concern is what is known as the recall bias where one group may recall events better than the other group. In the 2nd example, mothers who had abnormal babies may have spent lot of time reflecting on events that took place during the pregnancy but mothers who had normal babies may not have thought about this at any time. This leads to discrepancy of data in the two groups and can be a serious problem.

Case control studies are relatively cheap and take less time when compared with cohort studies. Also they allow the investigator to examine multiple exposures in one outcome. In bronchial carcinoma the investigator gets a chance to inquire about exposure to industrial dust and type of job in addition to smoking habits. Case control design is an ideal research method to study rare conditions such as congenital anomalies.

Selection of cases needs to be done carefully. One has to decide on case definition. If available, internationally accepted definitions are valuable and should be used (e.g. ACR criteria for SLE). If no such definition can be found, a clinically acceptable definition should be used. Results of a study can be of no interest simply due to poor case definition.

Equal importance should be given to the selection of controls. Controls need to be matched to resemble cases and sufficient care should be taken to exclude the presence of the particular disease under consideration. This can be difficult at times as asymptomatic patients may not like to undergo complicated and invasive procedures such as endoscopies and treadmill exercises. Furthermore, the type of control is also important. Controls can be selected from patients visiting hospitals (Hospital based controls), from neighborhood (Neighborhood controls) and community (Community controls) etc. The investigator must decide on the type of control suitable for his study.

Odd ratio (OR) is the usual statistics used in case control studies. It indicates the type of association existing between the outcome and exposure. OR of 1 indicates the lack of association between the two variables and values greater that 1 are considered risk factors. In contrast, values lesser than 1 are considered protective factors. OR is generally given with the $95 \%$ Confidence Intervals and this is required to decide whether the estimate is statistically significant. 


\section{Cross sectional design}

In this design, the investigator measures the exposure and outcome, both, in the same sitting.

\section{EXPOSURE $\longleftrightarrow$ OUTCOME}

\section{Examples}

1) Investigator measures BMI and the severity of knee osteoarthritis among postmenopausal women attending a rheumatology clinic.

2) Investigator measures FEV1 and mobility (Timed Get up and Go Test) of elderly women in a selected $\mathrm{MOH}$ area

Cross sectional studies are relatively easy, cheap and consume less time when compared with cohort studies. Furthermore, no "missing data" should be found as the exposure and outcome are measured simultaneously. However there are significant drawbacks in this study type.

It may not be possible to establish the causal relationship between the two variables measured in the study as one will not be able to tell the temporal relationship between the two. In the 1st example, it will be difficult to find out whether high BMI caused knee osteoarthritis or knee osteoarthritis caused less mobility and then high BMI.

\section{Clinical trials}

Clinical trials are conducted to estimate the effect of a chosen drug, procedure or life style intervention on the outcome of a disease. Unlike in observational studies, in clinical trials the investigator determines the type of the drug, procedure or intervention, dose and duration. In addition type of monitoring and care during the study period are determined by the investigator. Clinical trials mostly have only two groups of subjects and one group receives the test medication while other group is kept as the control group. The type of care given to the control group has to be determined by the investigator. Most of the time they are given a placebo drug which resembles the test drug (Figure 2).

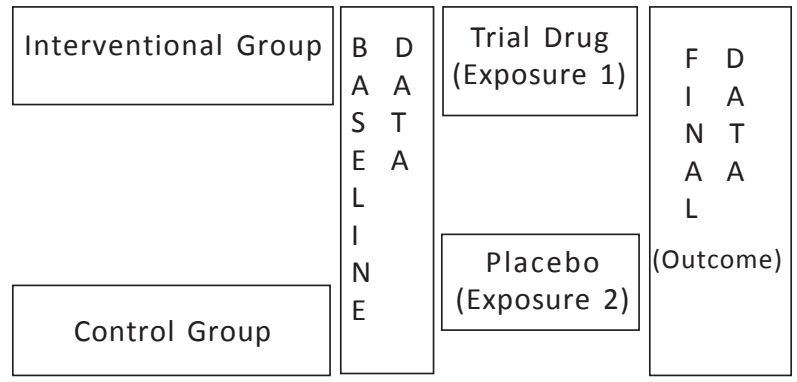

Figure 2. Study design of a clinical trial with two parallel groups.

\section{Examples}

1) Patients with congestive cardiac failure are randomized to two groups and one group is given spiranolactone in addition to the standard care. The other group is given a matching placebo in addition to the standard care. The investigator then counts the number of cardiac deaths that occur during the next two years in the two groups.

2) People with impaired fasting glucose are divided to two groups and one group is given intensive physical exercise program to follow and the other group is asked to follow a simple exercise program. The investigator then finds out the occurrence of diabetes in the two groups.

There are few key procedures to follow in clinical trials. They are done to eliminate various forms of biases and confounders that can compromise the validity of results. Randomisation of eligible subjects to study groups is one such step. Randomisation allows equal chances for all subjects to get into any group of the study, hence eliminates selection bias. More importantly, randomization ensures equal distribution of confounding factors between groups. If properly done, randomization helps to achieve prognostically similar study groups.

Blinding is the other key factor. Blinding makes the people concerned unaware of the type of care given to study subjects. Studies can be of Single-blind type where only patients are unaware of the type of care given to them. In Double-blind studies both patients as well as researches are unaware of the type of care given to study subjects. In addition there are critical groups that need to be blinded. They include parents (in paediatric studies), clinicians, data collectors and statisticians.

\section{Summary}

There are many study designs available for researches and the selection of the most suitable method is the first step in conducting a good research. Other key areas that ensure the validity of research outcome include proper selection of cases and controls and elimination of various forms of biases and confounders.

\section{Suggested further reading}

1. Epidemiology. 3rd Edition by Leon Gordis. Publisher: Elsevier Saunders.

2. Statistics at Square One. Swinscow TDV. Revised by Campbell MJ. BMJ Publishing Group 1997. 